\title{
Subcutaneous fat transplantation alleviates diet-induced glucose intolerance and inflammation in mice
}

\author{
Samantha L. Hocking ${ }^{1,2}$ • Rebecca L. Stewart ${ }^{1}$ - Amanda E. Brandon ${ }^{1}$ • \\ Eurwin Suryana $^{1} \cdot$ Ella Stuart $^{1} \cdot$ Emily M. Baldwin $^{1}$ • Ganesh A. Kolumam ${ }^{3}$. \\ Zora Modrusan $^{3}$ • Jagath R. Junutula ${ }^{3}$. Jenny E. Gunton ${ }^{4,5}$. \\ Michael Medynskyj ${ }^{6,7}$ - Sinead P. Blaber ${ }^{6,7}$ • Elisabeth Karsten ${ }^{6,7}$. \\ Benjamin R. Herbert $^{6,7}$ - David E. James ${ }^{1,8}$ • Gregory J. Cooney ${ }^{1,9}$ • \\ Michael M. Swarbrick ${ }^{1,4,10}$
}

Received: 30 October 2014 / Accepted: 13 March 2015 / Published online: 22 April 2015

(C) Springer-Verlag Berlin Heidelberg 2015

\begin{abstract}
Aims/hypothesis Adipose tissue (AT) distribution is a major determinant of mortality and morbidity in obesity. In mice, intra-abdominal transplantation of subcutaneous AT (SAT) protects against glucose intolerance and insulin resistance (IR), but the underlying mechanisms are not well understood. Methods We investigated changes in adipokines, tissuespecific glucose uptake, gene expression and systemic inflammation in male C57BL6/J mice implanted intra-abdominally with either inguinal SAT or epididymal visceral AT (VAT) and fed a high-fat diet (HFD) for up to 17 weeks.

Results Glucose tolerance was improved in mice receiving SAT after 6 weeks, and this was not attributable to differences in adiposity, tissue-specific glucose uptake, or plasma leptin or adiponectin concentrations. Instead, SAT transplantation prevented HFD-induced hepatic triacylglycerol accumulation and normalised the expression of hepatic gluconeogenic
\end{abstract}

Electronic supplementary material The online version of this article (doi:10.1007/s00125-015-3583-y) contains peer-reviewed but unedited supplementary material, which is available to authorised users.

Michael M. Swarbrick

m.swarbrick@garvan.org.au

1 Diabetes and Metabolism Division, Garvan Institute of Medical Research, 384 Victoria Street, Darlinghurst, 2010 Sydney, NSW, Australia

2 Department of Endocrinology, Royal North Shore Hospital, Sydney, NSW, Australia

3 Tumor Biology and Angiogenesis, Genentech Inc., South San Francisco, CA, USA

4 Centre for Diabetes, Obesity and Endocrinology, Westmead Millennium Institute, Westmead, NSW, Australia enzymes. Grafted fat displayed a significant increase in glucose uptake and unexpectedly, an induction of skeletal muscle-specific gene expression. Mice receiving subcutaneous fat also displayed a marked reduction in the plasma concentrations of several proinflammatory cytokines (TNF- $\alpha$, IL17, IL-12p70, monocyte chemoattractant protein-1 [MCP-1] and macrophage inflammatory protein $-1 \beta[$ MIP- $1 \beta]$ ), compared with sham-operated mice. Plasma IL-17 and MIP-1 $\beta$ concentrations were reduced from as early as 4 weeks after transplantation, and differences in plasma TNF- $\alpha$ and IL-17 concentrations predicted glucose tolerance and insulinaemia in the entire cohort of mice $(n=40)$. In contrast, mice receiving visceral fat transplants were glucose intolerant, with increased hepatic triacylglycerol content and elevated plasma IL-6 concentrations.

Conclusions/interpretation Intra-abdominal transplantation of subcutaneous fat reverses HFD-induced glucose

Westmead Clinical School, University of Sydney, Sydney, NSW, Australia

6 Biomolecular Frontiers Centre, Dept. of Chemistry and Biomolecular Science, Faculty of Science, Macquarie University, Sydney, NSW, Australia

7 Regeneus Ltd, Pymble, Sydney, NSW, Australia

8 Charles Perkins Centre, University of Sydney, Camperdown, NSW, Australia

9 St Vincent's Clinical School, UNSW Medicine, St Vincent's Hospital, Sydney, NSW, Australia

10 School of Medical Sciences, University of New South Wales Australia, Kensington, NSW, Australia 
intolerance, hepatic triacylglycerol accumulation and systemic inflammation in mice.

Keywords Adipose tissue - Body fat distribution - Fatty liver . Glucose intolerance · Inflammation · Insulin resistance · Intra-abdominal fat $\cdot$ Obesity $\cdot$ Subcutaneous fat $\cdot$ Visceral

$\begin{array}{ll}\text { Abbreviations } & \\ \text { ACC } & \text { Acetyl-CoA carboxylase } \\ \text { ACSL1 } & \begin{array}{l}\text { Acyl-CoA synthetase long-chain family } \\ \text { member 1 }\end{array} \\ \text { AT } & \text { Adipose tissue } \\ \text { DEXA } & \text { Dual-energy x-ray absorptiometry } \\ \text { EPI } & \text { Endogenous epididymal AT } \\ \text { FAS } & \text { Fatty acid synthase } \\ \text { GTT } & \text { Glucose tolerance test } \\ \text { GSEA } & \text { Gene Set Enrichment Analysis } \\ \text { G6Pase } & \text { Glucose 6-phosphatase } \\ \text { HFD } & \text { High-fat diet } \\ \text { iAUC } & \text { Incremental AUC } \\ \text { ING } & \text { Endogenous inguinal AT } \\ \text { IR } & \text { Insulin resistance } \\ \text { ITT } & \text { Insulin tolerance test } \\ \text { MCP-1/CCL2 } & \text { Monocyte chemotactic protein-1/ } \\ & \text { chemokine (C-C motif) ligand 2 } \\ \text { MEF } & \text { Myocyte enhancer factor } \\ \text { MIP-1 } \beta / C C L 4 & \text { Macrophage inflammatory protein-1 } \\ \text { beta/chemokine (C-C) ligand 4 } \\ \text { PEPCK } & \text { Phosphoenolpyruvate carboxykinase } \\ \text { RANTES/CCL5 } & \text { Regulated on activation, normal T cell } \\ & \text { expressed and secreted/ chemokine } \\ \text { (C-C) ligand 5 }\end{array}$

\section{Introduction}

Adipose tissue (AT) distribution is a major determinant of mortality and morbidity in obesity [1]. Specifically, accumulation of intra-abdominal or visceral AT (VAT) is associated with type 2 diabetes, dyslipidaemia and hypertension [2]. This relationship was originally attributed to increased NEFA flux from VAT into the portal circulation [3]. However, VAT makes only a minor contribution to portal NEFA concentrations [4], suggesting that other factors, such as adipokines [5], are also involved.

Independently of VAT, greater amounts of subcutaneous AT (SAT), particularly in the lower body, may protect against glucose intolerance, insulin resistance (IR), dyslipidaemia and atherosclerosis [6]. Gluteal-femoral SAT, for example, prevents lipotoxicity in non-adipose tissues by sequestering meal-derived NEFA [7, 8], and resists deleterious gene expression changes in obesity [9]. Understanding how different AT depots influence the risk of glucose intolerance, IR and dyslipidaemia may yield new therapeutic targets for metabolic disease [10].

Obesity and IR are characterised by chronic inflammation [11], with elevated proinflammatory cytokine production in AT [12]. Adipocyte hypertrophy in AT is accompanied by macrophage infiltration [13] and polarisation towards a classically activated proinflammatory (M1) type rather than 'alternatively' activated, antiinflammatory macrophages (M2) [14]. Proinflammatory cytokines impair insulin action both locally and in other tissues, such as liver [15]. AT from obese, insulinresistant humans also has a higher proinflammatory $\mathrm{T}$ cell content [16]. Anti-inflammatory treatment improves glycaemic control in humans with type 2 diabetes [17].

We and others have investigated the relationship between regional adiposity and metabolism by performing syngeneic AT transplantation in mice [18-22]. Intra-abdominal transplantation of epididymal AT was initially reported to improve glucose tolerance and reduce insulinaemia within 4-8 weeks [18]. In mice fed a high-fat diet (HFD) for 13 weeks, however, we found that intra-abdominal transplantation of inguinal AT, but not epididymal AT, improved glucose tolerance and reduced adiposity [19]. This did not occur when inguinal AT was transplanted subcutaneously. Our findings were confirmed by Tran et al [20], who also reported differences in AT glucose uptake between mice receiving intra-abdominal AT transplants and sham-operated mice, under euglycaemichyperinsulinaemic clamp conditions. More recently, intraabdominal transplantation of either inguinal or epididymal AT has been reported to reverse HFD-induced glucose intolerance and liver triacylglycerol accumulation within 4 weeks [21].

We aimed to address these discrepancies and identify the underlying mechanisms. While the beneficial effects of inguinal SAT transplantation on glucose tolerance are highly reproducible [19-22], epididymal AT transplantation is linked with both beneficial $[18,21]$ and negligible $[19,20]$ effects on metabolism, relative to sham-operated mice. Therefore, we performed time course studies to investigate the short- and long-term effects of inguinal subcutaneous $\rightarrow$ visceral (SubQ $\rightarrow$ Vis) and epididymal visceral $\rightarrow$ visceral (Vis $\rightarrow$ Vis) AT transplantation in HFD-fed mice. 
Table 1 Fat depot weights ${ }^{\mathrm{a}}$ in Sham, SubQ $\rightarrow$ Vis and Vis $\rightarrow$ Vis mice at 3,6 and 17 weeks after transplantation

\begin{tabular}{|c|c|c|c|c|}
\hline Variable & Sham & SubQ $\rightarrow$ Vis & Vis $\rightarrow$ Vis & $p$ \\
\hline \multicolumn{5}{|l|}{ At 3 weeks } \\
\hline$n$ & 8 & 6 & 8 & \\
\hline \multicolumn{5}{|l|}{ Endogenous fat } \\
\hline Epididymal & $506 \pm 61$ & $424 \pm 41$ & $437 \pm 44$ & 0.48 \\
\hline Inguinal & $453 \pm 30$ & $417 \pm 43$ & $424 \pm 37$ & 0.28 \\
\hline Intrascapular & $759 \pm 37$ & $747 \pm 48$ & $694 \pm 69$ & 0.43 \\
\hline Retroperitoneal & $129 \pm 19$ & $94 \pm 12$ & $105 \pm 15$ & 0.31 \\
\hline \multicolumn{5}{|l|}{ Transplanted fat } \\
\hline Recovered & - & $165 \pm 22$ & $224 \pm 13$ & 0.031 \\
\hline Initial & - & $195 \pm 15$ & $186 \pm 16$ & 0.71 \\
\hline \multicolumn{5}{|l|}{ At 6 weeks } \\
\hline$n$ & 10 & 7 & 10 & \\
\hline \multicolumn{5}{|l|}{ Endogenous fat } \\
\hline Epididymal & $874 \pm 96$ & $690 \pm 102$ & $590 \pm 63$ & 0.068 \\
\hline Inguinal & $670 \pm 67$ & $584 \pm 73$ & $466 \pm 38$ & 0.056 \\
\hline Intrascapular & $982 \pm 72$ & $894 \pm 94$ & $740 \pm 44^{*}$ & 0.045 \\
\hline Retroperitoneal & $253 \pm 36$ & $194 \pm 48$ & $164 \pm 24$ & 0.18 \\
\hline \multicolumn{5}{|l|}{ Transplanted fat } \\
\hline Recovered & - & $147 \pm 11$ & $283 \pm 86$ & 0.0004 \\
\hline Initial & - & $252 \pm 12$ & $225 \pm 14$ & 0.18 \\
\hline \multicolumn{5}{|l|}{ At 17 weeks } \\
\hline$n$ & 8 & 7 & 9 & \\
\hline \multicolumn{5}{|l|}{ Endogenous fat } \\
\hline Epididymal & $1,266 \pm 204$ & $1,120 \pm 105$ & $1,109 \pm 97$ & 0.69 \\
\hline Inguinal & $986 \pm 134$ & $770 \pm 77$ & $905 \pm 95$ & 0.40 \\
\hline Intrascapular & $1,125 \pm 172$ & $903 \pm 55$ & $1,002 \pm 92$ & 0.46 \\
\hline Retroperitoneal & $409 \pm 65$ & $358 \pm 40$ & $422 \pm 51$ & 0.70 \\
\hline \multicolumn{5}{|l|}{ Transplanted fat } \\
\hline Recovered & - & $160 \pm 27$ & $587 \pm 75$ & 0.0003 \\
\hline Initial & - & $244 \pm 18$ & $239 \pm 14$ & 0.84 \\
\hline
\end{tabular}

All data are shown as means \pm SEM

${ }^{\mathrm{a}}$ Fat depot weights are given in $\mathrm{mg}$

${ }^{*} p<0.05$ vs Sham

\section{Methods}

Animals and surgery Six-week-old male C57BL6/J mice were obtained from Australian Bioresources (Moss Vale, NSW, Australia) and housed as described previously [23]. Studies were approved by the Garvan Institute/St Vincent's Hospital Animal Experimentation Ethics Committee, and were performed according to guidelines set by the National Health and Medical Research Council of Australia.

Inguinal and epididymal AT depots were dissected out from donors and transplanted into anaesthetised recipient littermates via a midline incision. AT was sutured onto the peritoneal surface of the anterior abdominal wall using Vicryl 4-0 sutures (Ethicon, Somerville, NJ, USA). The incision was closed with wound clips. Sham-operated mice received identical surgical treatment without transplant. After regaining their pre-surgical weight, mice were provided ad libitum with either chow (8\% of energy from fat, Gordon's Specialty Stock Feeds, Yanderra, NSW, Australia) or HFD (45\% of energy from fat, based on D12451, Research Diets, New Brunswick, NJ, USA). At the end of the experiment, mice were killed by cervical dislocation.

Body composition, respirometry and food intake Body composition was measured by dual-energy $\mathrm{x}$-ray absorptiometry (DEXA; Lunar PIXImus, GE Medical Systems, New York, NY, USA) under isoflurane anaesthesia. Twelve weeks 
Fig. 1 Whole-body effects of intra-abdominal AT mice ( $n=6-8 /$ group) were studied for 3 weeks, 27 mice $(n=7-10$ / group) for 6 weeks and 24 mice ( $n=7-9$ /group) for 17 weeks. (b) Body weight: Sham, SubQ $\rightarrow$ Vis and Vis $\rightarrow$ Vis mice are indicated by black circles, white circles and black squares, respectively. (c) Fat mass; (d) lean mass; (c, d) Sham, SubQ $\rightarrow$ Vis and Vis $\rightarrow$ Vis mice are indicated by black, white and striped columns, respectively ( $n=14-19 /$ group at 6 weeks and $n=7-9$ /group at 13 weeks). (e-h) Glucose tolerance at 3, 6, 10 and 14 weeks, respectively; symbols are as for Fig. 1b. (i) iAUC at each time point. ${ }^{*} p<0.05$ vs Sham, ${ }^{\dagger} p<0.05$ and ${ }^{\dagger \dagger} p<0.01$ vs Vis $\rightarrow$ Vis $(n=6-8, n=14-19, n=7-9$ and $n=7-9$ /group at 3, 6, 10 and 14 weeks, respectively); bar at 7 weeks; symbols are as for vs Vis $\rightarrow$ Vis and $* * p<0.01$ for $\mathrm{SubQ} \rightarrow$ Vis vs Sham mice. (k) ITT expressed relative to baseline; ${ }^{*} p<0.05$ for Sham vs Vis $\rightarrow$ Vis mice. EE, energy expenditure; IPGTT, intraperitoneal GTT; T, transplantation transplantation. (a) Cohorts: 22 colour is as for Fig. 1c, d. (j) ITT Fig. $1 \mathrm{~b} ;{ }^{\dagger} p<0.05$ for SubQ $\rightarrow$ Vis

a $\prod_{\text {Week }} \frac{\left.\right|_{30} ^{\text {HFD }}}{30}$
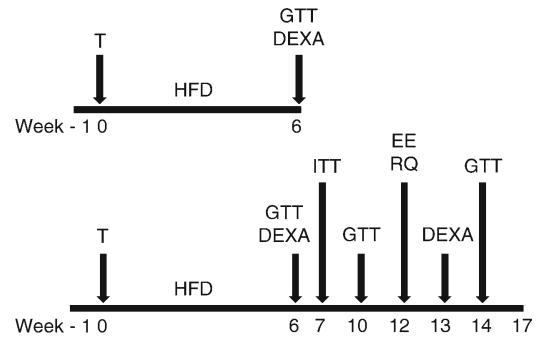

b
C
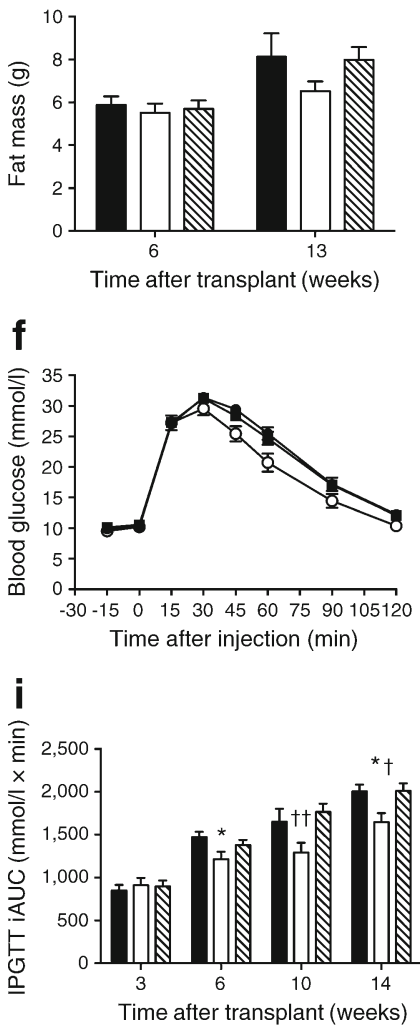

d
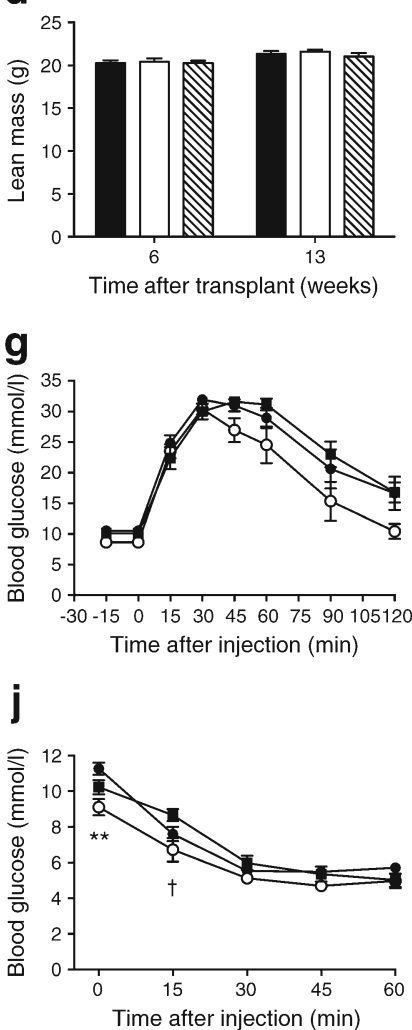

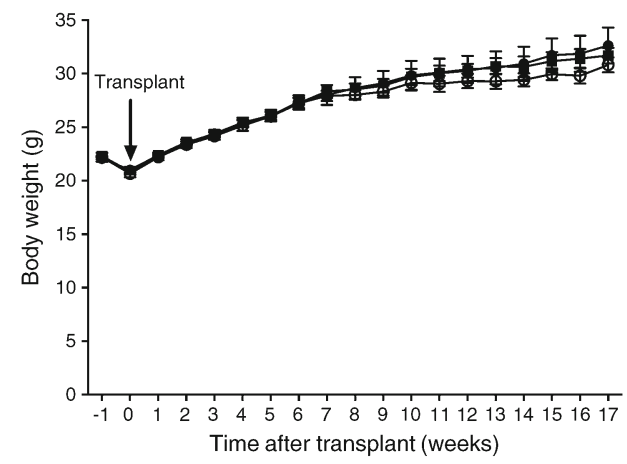

e

h
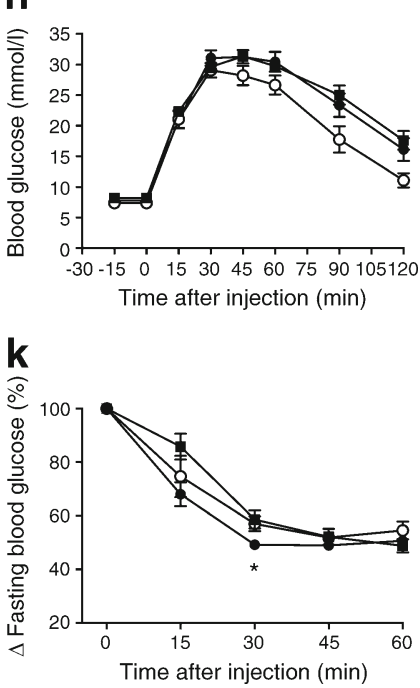



after transplantation, food intake, energy expenditure and RQ were measured as described previously [23].

Glucose and insulin tolerance tests Intraperitoneal glucose tolerance tests (GTTs; $2 \mathrm{~g} / \mathrm{kg}$ ) were performed in conscious 6-h-fasted mice. For insulin tolerance tests (ITTs), mice were fasted for $4 \mathrm{~h}$ before injection of $0.75 \mathrm{U} / \mathrm{kg}$ body weight i.p. (Actrapid, Novo Nordisk, Baulkham Hills, NSW, Australia). Blood glucose concentrations were measured using an AccuChek meter (Roche Diagnostics, Castle Hill, NSW, Australia).

For measurement of tissue-specific glucose uptake, 6-hfasted mice were injected with $1.5 \mathrm{~g} / \mathrm{kg}$ glucose containing tracer amounts of $\left[\mathrm{U}-{ }^{14} \mathrm{C}\right]$ glucose $(370,000 \mathrm{~Bq} /$ mouse $)$ and $\left[{ }^{3} \mathrm{H}-2\right]$ deoxyglucose $(37,000 \mathrm{~Bq} /$ mouse; Perkin-Elmer, Glen

Fig. 2 Effects of transplantation on grafted AT. (a) Relative changes in graft mass. SubQ $\rightarrow$ Vis mice $(n=6-7 /$ cohort) are indicated by white circles and Vis $\rightarrow$ Vis mice ( $n=8-10$ /cohort) by black squares. (b) Adipocyte area in grafts at 3 weeks; $p<0.0001$ for difference, $\chi^{2}$ test. (c) Adipocyte area in grafts at 6 weeks; $p=0.044, \chi^{2}$ test. (d) Adipocyte area in grafts at 17 weeks; $p<0.0001, \chi^{2}$ test. $(\mathbf{e}-\mathbf{g}) \mathrm{SubQ} \rightarrow$ Vis grafts at 3, 6 and 17 weeks, respectively. Scale bars, $100 \mu \mathrm{m}$. (h-j) Vis $\rightarrow$ Vis grafts at 3, 6 and 17 weeks, respectively. Scale bars, $100 \mu \mathrm{m}$. (k) Plasma leptin concentrations. Sham, SubQ $\rightarrow$ Vis and Vis $\rightarrow$ Vis mice are indicated by black, white and striped columns, respectively $(n=8$, $n=5-6$ and $n=8-9$, respectively, for Sham, SubQ $\rightarrow$ Vis and Vis $\rightarrow$ Vis mice at each time point). Effect of time: $p<0.0001$; effect of group: $p=0.17$; two-way ANOVA. (I) Plasma adiponectin concentrations; bars are as for Fig. $2 \mathrm{k}$; effect of time: $p=0.055$; effect of group: $p=0.63$ 


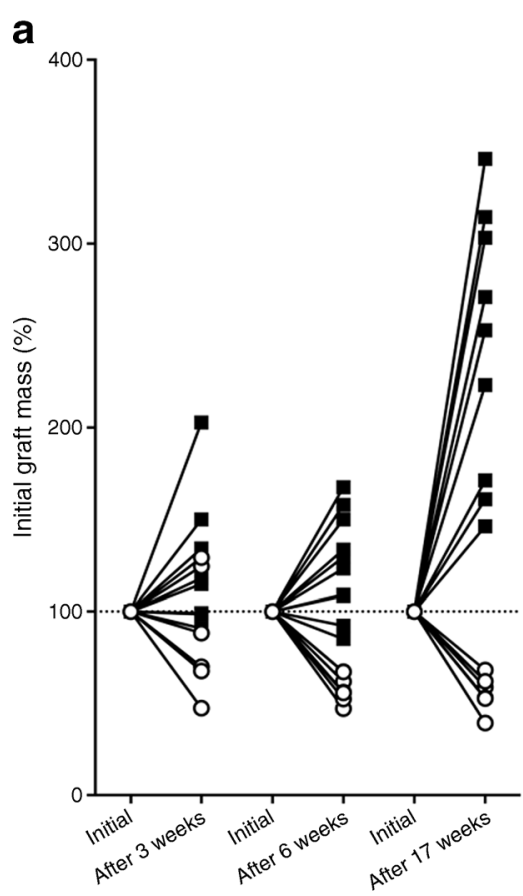

At 3 weeks
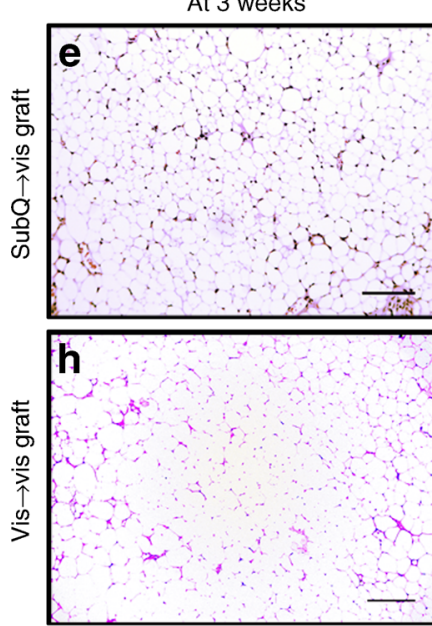

k

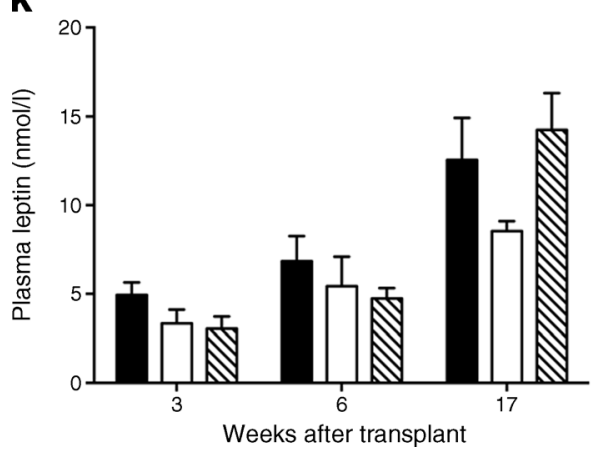

Waverley, VIC, Australia). Tail blood was collected into heparinised tubes (Microvette CB300LH, Sarstedt, Mawson
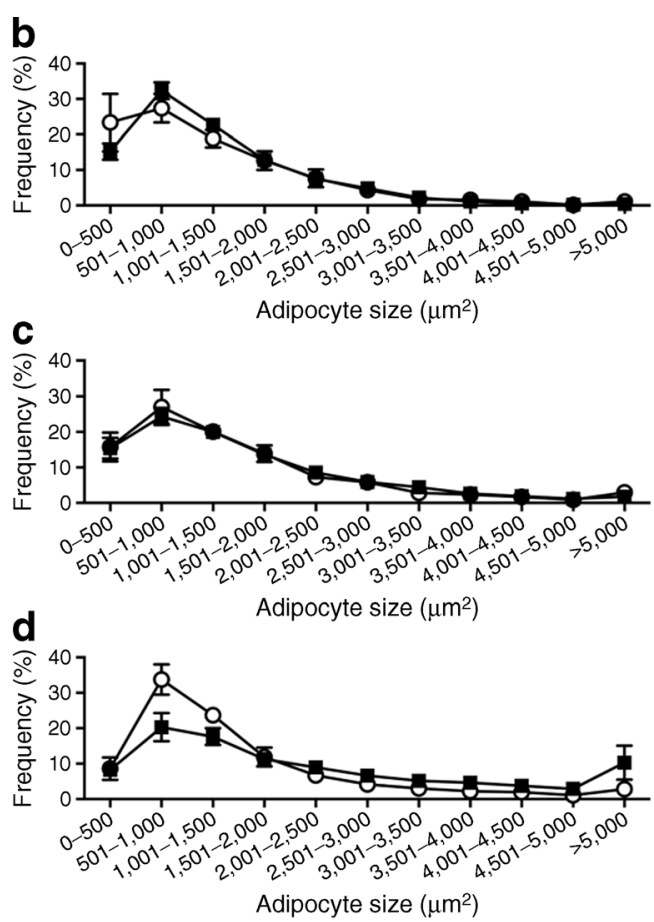

At 6 weeks

At 17 weeks
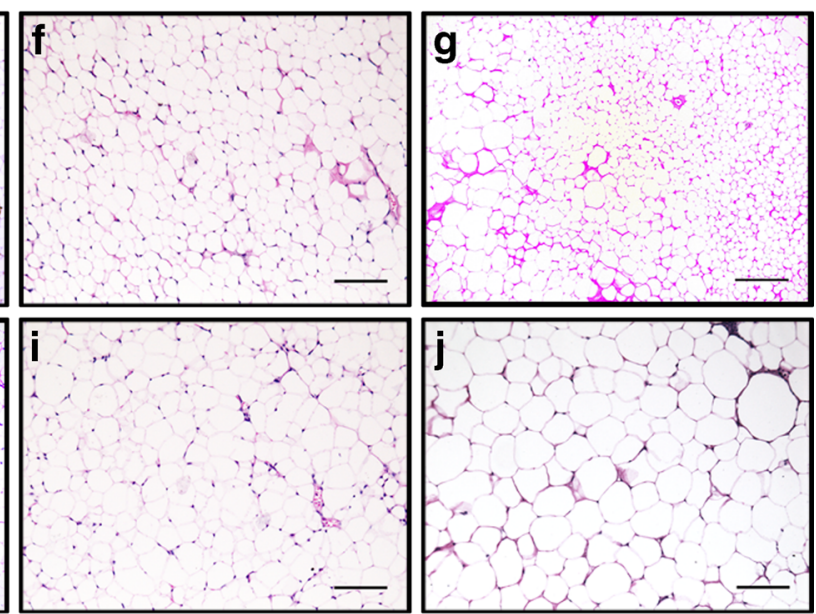

I

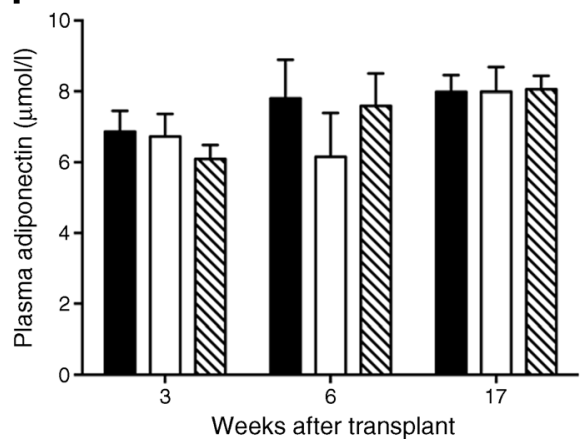

Lakes, SA, Australia), and plasma was separated by centrifuging at 2,000×g for $5 \mathrm{~min}$. Glucose uptake in skeletal muscle 
and individual AT depots was normalised for the specific activity of glucose in plasma as described previously [24].

AT adipocyte sizing and immunohistochemistry Tissues were fixed in $3.7 \%$ (vol./vol.) formaldehyde, paraffin-embedded, cut into $4 \mu \mathrm{m}$ sections and stained with haematoxylin and eosin. Adipocyte size was determined in three representative fields from each section (100-150 adipocytes/field) using ImageJ software (National Institutes of Health, USA). Adipocyte size distribution was determined for each depot by assigning adipocytes to $500 \mu \mathrm{m}^{2}$ bins. Differences between groups were determined by $\chi^{2}$ test. Graft apoptosis was assessed by cleaved caspase-3 immunohistochemistry [25].

Biochemical methods Tissue triacylglycerol and glycogen content, and plasma insulin and NEFA concentrations were measured as described [23]. Plasma leptin and adiponectin concentrations were measured by ELISA (Crystal Chem, Downers Grove, IL, USA and R\&D Systems, Minneapolis, MN, USA, respectively). Plasma lactate was measured using a YSI 2300 Analyzer (YSI Life Sciences, Yellow Springs, OH, USA).

Microarrays Tissues were obtained in our previous 13 week study [19], which included mice receiving subcutaneous inguinal AT in the dorsal subcutaneous space (SubQ $\rightarrow$ SubQ). RNA was extracted from the following depots $(n=5$ of each): SubQ $\rightarrow$ Vis and SubQ $\rightarrow$ SubQ grafts, endogenous inguinal SAT from SubQ $\rightarrow$ Vis mice (SubQ $\rightarrow$ Vis ING), endogenous epididymal AT from $\mathrm{SubQ} \rightarrow$ Vis mice $(\mathrm{SubQ} \rightarrow$ Vis EPI) and inguinal SAT from a sham-operated mouse (Sham ING).

cDNA preparation and array hybridisation were performed according to the manufacturer's instructions (Affymetrix Mouse Genome 430 2.0, Santa Clara, CA, USA). Gene expression differences $>$ twofold were analysed using Genepattern software [26]. Gene Set Enrichment Analysis (GSEA) was performed using the $\mathrm{c} 3$ all and $\mathrm{c} 5$ collections in the Molecular Signatures Database (MSigDB) [27, 28]. Results were deposited in a public functional genomics data repository (Gene Expression Omnibus, www.ncbi.nlm.nih. gov/geo).

Immunoblotting Tissues were homogenised in RIPA buffer $(65 \mathrm{mmol} / \mathrm{l} \mathrm{Tris} \cdot \mathrm{HCl}, 150 \mathrm{mmol} / \mathrm{l} \mathrm{NaCl}, 5 \mathrm{mmol} / \mathrm{l}$ EDTA, $1 \% \mathrm{NP}-40$ [vol./vol.], $0.5 \%$ sodium deoxycholate [wt/vol.], $0.1 \%$ SDS [wt/vol.] and $10 \%$ glycerol [vol./vol.], $\mathrm{pH} 7.4$ ) containing $1 \mathrm{mg} / \mathrm{l}$ aprotinin, $1 \mathrm{mg} / 1$ leupeptin, $10 \mathrm{mmol} / 1$ $\mathrm{NaF}, 1 \mathrm{mmol} / 1 \mathrm{Na}_{3} \mathrm{VO}_{4}$ and $1 \mathrm{mmol} / \mathrm{l}$ PMSF. Cleared lysates $(20 \mu \mathrm{g}$ protein) were electrophoresed in $10 \%$ polyacrylamide gels and transferred to PVDF membranes (GE Healthcare, Buckinghamshire, UK). Antibodies for stearoyl-CoA desaturase-1 (SCD1; 2438), fatty acid synthase (FAS; 3180), acetyl-CoA carboxylase (ACC; 36625 ) and acyl-CoA synthetase long-chain family member 1 (ACSL1; 4047) were obtained from Cell Signaling Technologies (Danvers, MA, USA). The sterol regulatory element-binding protein (SREBP1) antibody (MS1207P1ABX) was obtained from Thermo Scientific (Scoresby, VIC, Australia). Glucose 6-phosphatase (G6Pase) and 14-3-3 (sc-25840 and sc-629, respectively) antibodies were supplied by Santa Cruz Biotechnology (Santa Cruz, CA, USA). Appropriate HRP-conjugated secondary antibodies were obtained from Jackson ImmunoResearch (West Grove, PA, USA). Densitometry was performed using ImageJ software.

Cytokine profiling Plasma was collected at 4 and 10 weeks post-transplantation. Freshly thawed plasma was filtered using sterile Ultrafree-MC GV centrifuge filters (Millipore, Kilsyth, VIC, Australia) and cytokine concentrations were determined using a Bio-Plex Pro Mouse Cytokine Assay (M60-009RDPD) and a Bio-Plex 200 instrument (BioRad, Hercules, CA, USA). The intra-assay CV for all cytokines was $>5 \%$.

Statistical analysis All data are presented as mean \pm SEM. Glucose excursions during the GTT were expressed as incremental AUC (iAUC), calculated using Microsoft Excel. Differences between groups were analysed by Student's $t$ test, one-way or two-way ANOVA (or their non-parametric equivalents) using GraphPad Prism (La Jolla, CA, USA). Multiple comparisons were corrected using the Holm-Sidak (parametric) or Dunn's (non-parametric) tests. Relationships between continuous variables were assessed using Pearson's or Spearman correlations, as appropriate. A two-sided $p<0.05$ was considered significant.

\section{Results}

Glucose tolerance in SubQ $\rightarrow$ Vis mice was improved from 6 weeks post-transplantation We previously reported beneficial effects of SubQ $\rightarrow$ Vis transplantation at $12-13$ weeks [19]. Here, we studied HFD-fed Sham, SubQ $\rightarrow$ Vis and Vis $\rightarrow$ Vis mice for 3, 6 and 17 weeks (Fig. 1a). AT transplantation did not significantly affect body weight (Fig. 1b), fat mass (Fig. 1c) or lean mass (Fig. 1d). Masses of AT depots at 3, 6, and 17 weeks after transplantation are shown in Table 1 . No significant differences in energy expenditure, RQ or food intake were observed (Electronic supplementary material [ESM] Table 1).

Glucose tolerance was sustainably improved in SubQ $\rightarrow$ Vis mice from 6 weeks onwards (Fig. 1e-i). At 7 weeks after transplantation, SubQ $\rightarrow$ Vis mice had the lowest basal 
Fig. 3 Radiolabelled GTTs. (a) Endogenous and grafted AT masses. Results from chow (vertical striped bars, $n=10$ ), HFD Sham (black bars, $n=12$ ), HFD SubQ $\rightarrow$ Vis (white bars, $n=8)$ and Vis $\rightarrow$ Vis (diagonally striped bars, $n=10$ ) mice are shown. $* * p<0.01$ and *** $p<0.001$ vs Sham, ${ }^{\dagger} p<0.05$ vs Vis $\rightarrow$ Vis mice. (b) Plasma glucose concentrations. Results from chow (white squares, $n=10$ ), HFD Sham (black circles, $n=12$ ), HFD SubQ $\rightarrow$ Vis (white circles, $n=8$ ) and HFD Vis $\rightarrow$ Vis (black squares, $n=10$ ) mice are shown. (c) Plasma glucose iAUC; bars are as for Fig. $3 \mathrm{a} ;{ }^{*} p<0.05$ vs Sham; ${ }^{\dagger} p<0.05$ vs Vis $\rightarrow$ Vis. (d) Plasma insulin concentrations; symbols are as for Fig. 3b; ${ }^{*} p<0.05$, ANOVA. (e) Plasma insulin AUC; bars are as for Fig. 3a; overall $p=0.092$, Kruskal-Wallis test. (f) Plasma NEFA concentrations; symbols are as for Fig. 3b; effect of time: $p<0.0025$; effect of group: $p=0.35$, two-way repeated measures ANOVA. (g) Immunoblot for hepatic gluconeogenic enzymes PEPCK and G6Pase. Pooled samples were used ( $n=8-12$ /group). PEPCK and G6Pase expression was normalised to 14-3-3 (loading control). (h) Skeletal muscle and AT glucose uptake; bars are as for Fig. $3 \mathrm{a}$; ${ }^{*} p<0.05, * * p<0.01$, $* * * p<0.001$ and $* * * * p<0.0001$ vs Sham; ${ }^{\dagger} p<0.01$ and ${ }^{\dagger \dagger} p<0.001$ for graft vs endogenous AT. DPM, disintegrations per minute; IPGTT, intraperitoneal GTT a

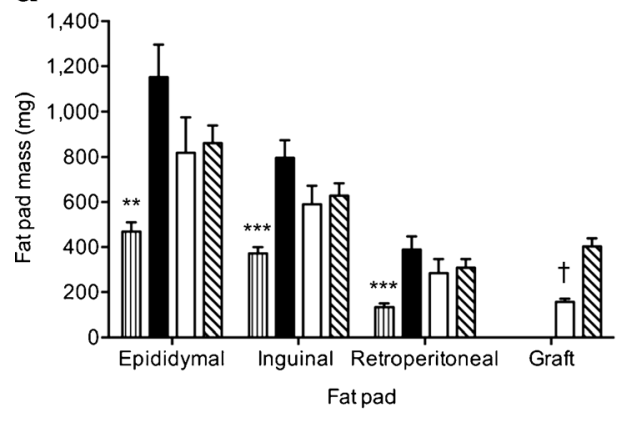

C

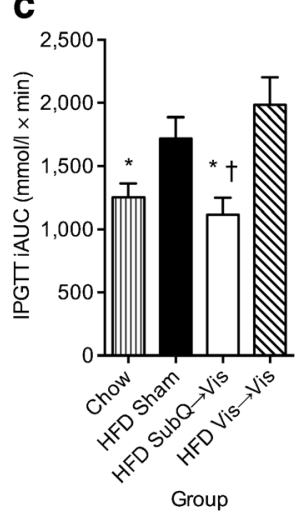

f

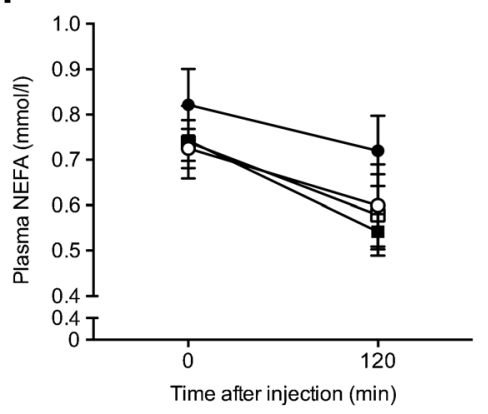

d
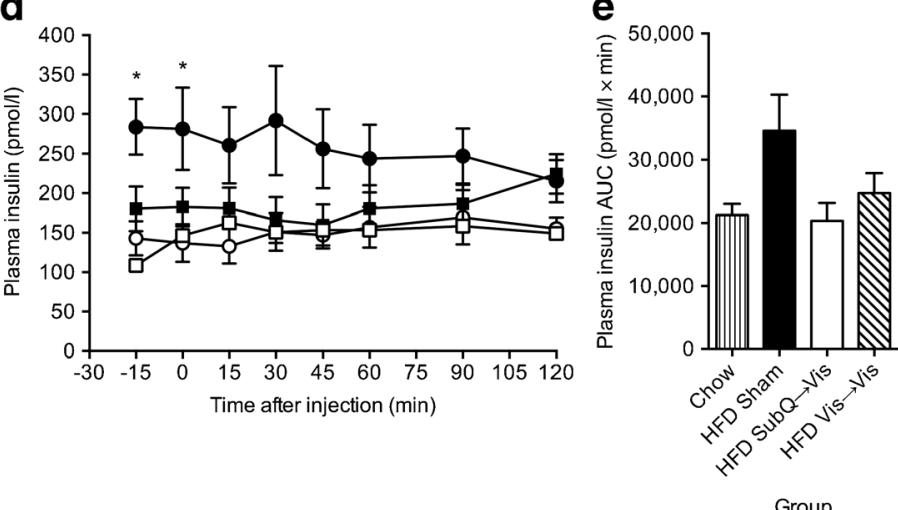

g

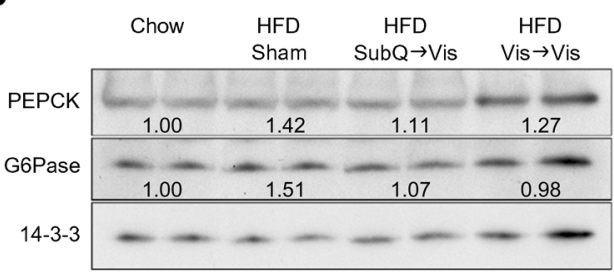

b

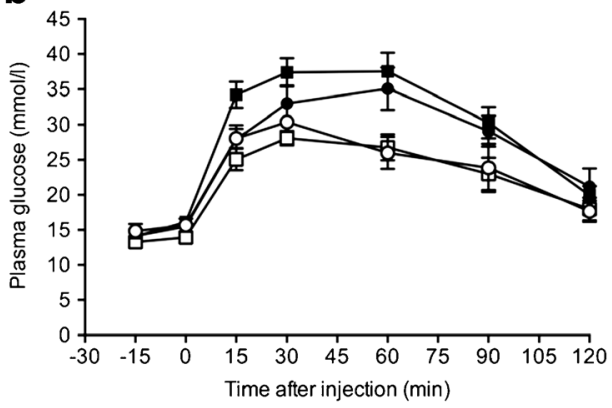

h 
a



\section{Sham mouse}

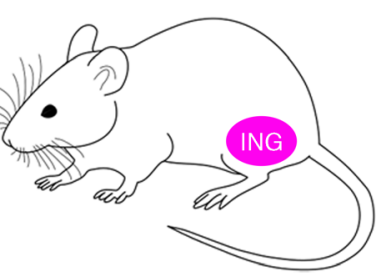

SubQ $\rightarrow$ SubQ mouse

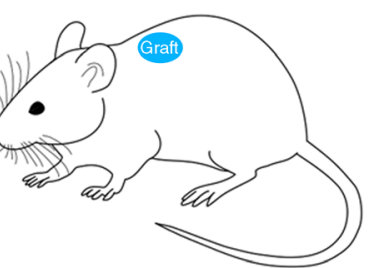

b



$-3.0-2.5-2.0-1.5-1.0-0.50 .0 \quad 0.51 .01 .52 .02 .53 .0$ Normalised expression

\section{C}

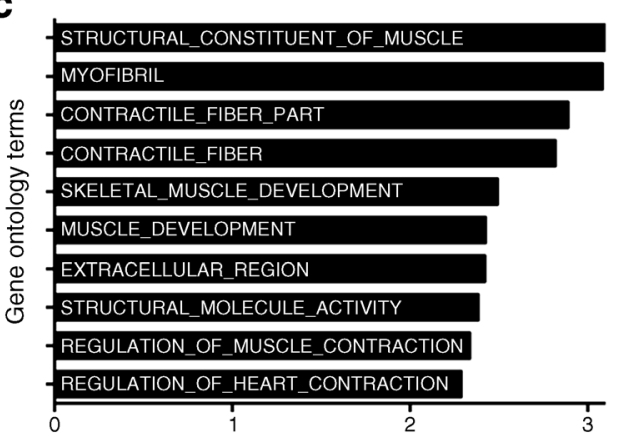

Normalised enrichment score

e

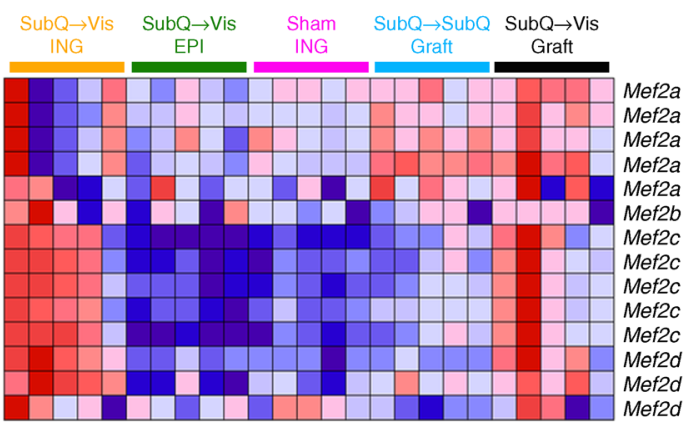

d



Normalised enrichment score

f

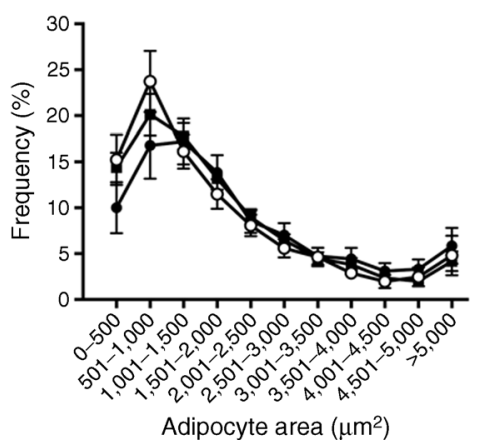


4 Fig. 4 Gene expression analysis of grafted and endogenous AT. (a) Endogenous and transplanted AT depots studied. (b). Skeletal musclespecific genes, including MEF2A targets, were induced in both the grafted and endogenous inguinal AT of SubQ $\rightarrow$ Vis mice. Genes displaying a $>$ fivefold increase in expression (relative to SubQ $\rightarrow$ SubQ grafts) are listed. Genes increased in both SubQ $\rightarrow$ Vis grafts and the endogenous inguinal SAT of SubQ $\rightarrow$ Vis mice (SubQ $\rightarrow$ Vis ING) are

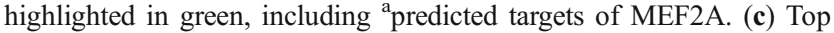
ten sets of genes over-represented in SubQ $\rightarrow$ Vis grafts vs SubQ $\rightarrow$ SubQ grafts. (d) Top ten sets of genes over-represented in endogenous inguinal AT of SubQ $\rightarrow$ Vis vs Sham mice. (e) MEF2 subtype expression in transplanted and endogenous AT. MEF2C and MEF2D were selectively expressed in the endogenous inguinal depot of SubQ $\rightarrow$ Vis mice $(q<0.005$ vs SubQ $\rightarrow$ Vis EPI or Sham ING). (f) Adipocyte size in the endogenous inguinal AT of Sham, SubQ $\rightarrow$ Vis and Vis $\rightarrow$ Vis mice. Sham, SubQ $\rightarrow$ Vis and Vis $\rightarrow$ Vis mice are indicated by black circles, white circles and black squares, respectively ( $n=7-9$ per group), $p<0.0001, \chi^{2}$ test

Improved glucose tolerance in $\mathrm{SubQ} \rightarrow$ Vis mice does not involve altered skeletal muscle or endogenous AT glucose uptake Next, we investigated tissue-specific glucose uptake in chow-fed mice and HFD-fed Sham, SubQ $\rightarrow$ Vis and Vis $\rightarrow$ Vis mice at 13 weeks after transplantation. HFD feeding increased adiposity (Fig. 3a), worsened glucose tolerance (Fig. 3b, c) and increased fasting insulinaemia (Fig. 3d).

$\mathrm{SubQ} \rightarrow$ Vis mice were completely protected against HFDinduced glucose intolerance (Fig. 3b, c); and transplantation of either type of AT significantly reduced fasting insulinaemia (Fig. 3d, e). Suppression of NEFA concentrations was used as a surrogate measure of AT insulin sensitivity: fasting and 120 min NEFA concentrations tended to be higher in HFD-fed Sham mice compared with the other groups (Fig. 3f). SubQ $\rightarrow$ Vis transplantation also reversed HFD-induced expression of the hepatic gluconeogenic enzymes phosphoenolpyruvate carboxykinase (PEPCK) and G6Pase (Fig. 3g).

Glucose uptake into quadriceps muscle and endogenous AT (epididymal, inguinal, retroperitoneal, intrascapular brown) was blunted by HFD feeding but did not differ among HFD-fed Sham, SubQ $\rightarrow$ Vis and Vis $\rightarrow$ Vis mice (Fig. 3h). Grafted SAT and VAT displayed similar glucose uptake; however, when compared with their endogenous depots, glucose uptake was increased by 2.8 -fold and 2.0-fold in SubQ $\rightarrow$ Vis and Vis $\rightarrow$ Vis grafts, respectively. Chronically increasing AT glucose uptake increases lactate release in the fed state and improves insulin sensitivity [29]: SubQ $\rightarrow$ Vis mice tended to have the highest postprandial plasma lactate concentrations at 17 weeks (ESM Fig. 2). Tracer incorporation into glycogen and triacylglycerol is shown in ESM Fig. 3.

\section{Grafted and endogenous subcutaneous depots of SubQ $\rightarrow$} Vis mice display an induction of skeletal muscle-specific genes To better understand the metabolic effects of SubQ $\rightarrow$ Vis transplantation, we compared gene expression in SAT implanted either intra-abdominally (SubQ $\rightarrow$ Vis) or subcutaneously (SubQ $\rightarrow$ SubQ) [19] (Fig. 4a). Twenty-eight genes displayed a $>$ fivefold increase in expression in SubQ $\rightarrow$ Vis compared with SubQ $\rightarrow$ SubQ grafts (Fig. 4b); many were cytoskeletal components of skeletal muscle (Fig. 4c). GSEA identified a common transcription factor regulating 12 of these genes: myocyte enhancer factor 2A (MEF2A). MEF2 transcription factors have established roles in myogenesis, muscle metabolism, nervous system development/repair and more recently, immune response [30].

Remarkably, expression of many of these genes was also induced in the endogenous inguinal AT of SubQ $\rightarrow$ Vis mice, relative to Sham-operated mice (Fig. 4b). GSEA of endogenous inguinal AT revealed enrichment for genes related to RNA processing and immune responses (Fig. 4d). Mef $2 c$ and $M e f 2 d$ were selectively induced in endogenous inguinal AT of SubQ $\rightarrow$ Vis mice (Fig. 4e); and SubQ $\rightarrow$ Vis mice had smaller adipocytes in this depot (Fig. 4f).

SubQ $\rightarrow$ Vis transplantation protects against HFDinduced hepatic triacylglycerol accumulation AT transplantation has been previously reported to reduce HFDinduced hepatic triacylglycerol accumulation [21, 22]. At 17 weeks, hepatic triacylglycerol content was reduced in $\mathrm{SubQ} \rightarrow$ Vis mice, relative to Vis $\rightarrow$ Vis mice, but no differences were observed at 6 weeks, when glucose tolerance was first improved (Fig. 5a). Hepatic glycogen content was unaffected (Fig. 5b).

Next, we measured the levels of the insulin-regulated lipogenic factor SREBP1 and its target genes (Fig. 5c-h). SREBP1 content and that of its target SCD1 tended to be highest in Vis $\rightarrow$ Vis mice (Fig. 5e, f), but the differences were not statistically significant. Levels of other SREBP targets, FAS, ACC and ACSL1, were not altered (Fig. 5g, h). Quadriceps muscle triacylglycerol content was significantly elevated in SubQ $\rightarrow$ Vis mice at 6 weeks after transplantation, but not at 17 weeks (Fig. 5i). Transplantation did not affect muscle glycogen content (Fig. 5j).

We also investigated gene expression using PCR arrays (ESM Fig. 4). Stat3 mRNA was elevated in livers from $\mathrm{SubQ} \rightarrow$ Vis mice compared with Vis $\rightarrow$ Vis mice $(p<0.05)$, and tended to be higher than in livers from Sham mice ( $p=0.056)$. Relative to Sham mice, Socs3, Adipor 2, Lpl and Foxa 2 mRNA were all significantly downregulated in livers from Vis $\rightarrow$ Vis mice. In human hepatocarcinoma cells, ADIPOR2 expression may be negatively regulated by SREBP1 [31].

$\mathrm{SubQ} \rightarrow$ Vis transplantation reduced circulating markers of inflammation Plasma cytokine profiling was also performed at 4 and 10 weeks after transplantation (Fig. 6 \& ESM Tables 2 and 3). SubQ $\rightarrow$ Vis transplantation significantly attenuated HFD-induced increases in plasma concentrations of TNF- $\alpha$, IL-6 and IL-17 (Fig. 6a-c). Plasma IL-6 concentrations were uniquely elevated in $\mathrm{Vis} \rightarrow \mathrm{Vis}$ mice at 10 weeks 

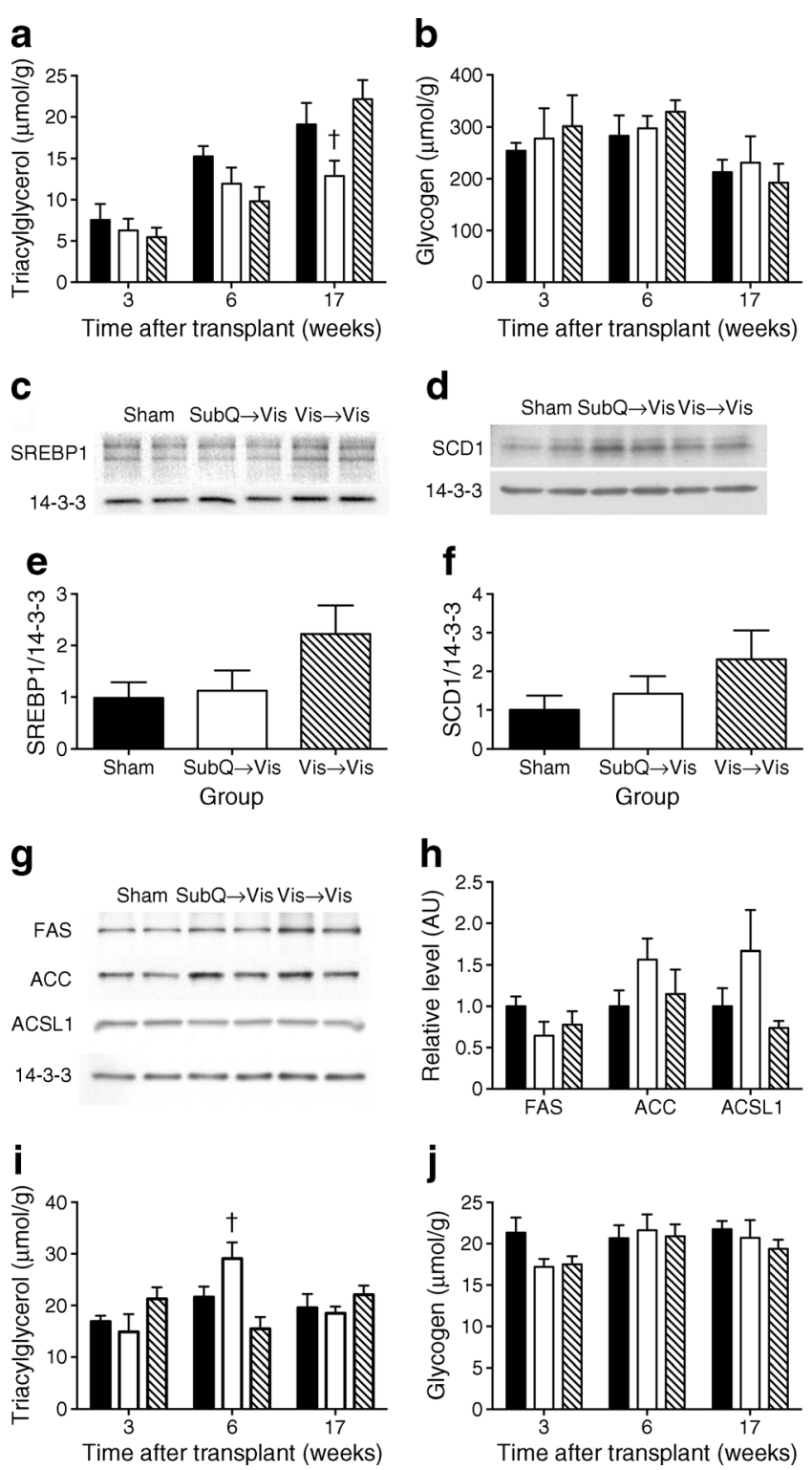

Fig. 5 SubQ $\rightarrow$ Vis transplantation protects mice against HFD-induced hepatic triacylglycerol accumulation. (a, b) Hepatic triacylglycerol (a) and glycogen (b) content at 3, 6 and 17 weeks after transplantation; ${ }^{\dagger} p<0.05$ vs Vis $\rightarrow$ Vis. (c, d) Immunoblots for hepatic SREBP1 (c) and hepatic SCD1 (d) levels at 17 weeks after transplantation. (e, f) Relative levels of SREBP 1 (e) and SCD1 (f); $p=0.12$ (e) and $p=0.24$ (f), ANOVA. (g) Immunoblot for hepatic FAS, ACC and ACSL1 levels at 17 weeks after transplantation. (h) Relative FAS, ACC and ACSL1 levels; $p=0.33$, 0.32 and 0.70 for differences in FAS, ACC and ACSL1 content, ANOVA. $(\mathbf{i}, \mathbf{j})$ Quadriceps muscle triacylglycerol (i) and glycogen (j) content at 3, 6 and 17 weeks after transplantation; ${ }^{\dagger} p<0.05$ vs Vis $\rightarrow$ Vis. In all cases, Sham ( $n=8-10$ /group), SubQ $\rightarrow$ Vis $(n=6-8 /$ group) and Vis $\rightarrow$ Vis ( $n=8-10$ /group) mice are indicated by black, white and striped columns, respectively. AU, arbitrary units

after transplantation (Fig. 6b). HFD feeding is associated with increased plasma concentrations of IL-12p70 and the chemokines monocyte chemotactic protein-1/chemokine (CC) motif) ligand 5 (MCP-1/CCL5) and macrophage inflammatory protein-1 beta/chemokine (C-C) ligand 4 (MIP-1 $\beta /$ CCL4) [32-34]; all were significantly reduced in SubQ $\rightarrow$
Vis mice relative to Sham mice (Fig. 6d-f). Concentrations of the Th1 chemokine RANTES/CCL5 (regulated on activation, normal $\mathrm{T}$ cell expressed and secreted/chemokine (C-C) ligand 5) were suppressed in SubQ $\rightarrow$ Vis and Vis $\rightarrow$ Vis mice compared with Sham mice (Fig. 6g).

Th2 cells promote anti-inflammatory (M2) macrophage polarisation via the production of IL-4, IL-10 or IL-13 [35]. Plasma IL-10 and IL-4 concentrations were lower in SubQ $\rightarrow$ Vis mice than in Sham mice at 10 weeks (Fig. 6h, i). Transplantation of both types of AT suppressed plasma concentrations of IL-13, and those of the Th1 cytokines IL-5 and IL-2 (Fig. 6j-1).

Next, we examined relationships between the above cytokines and either the response to a GTT (iAUC GTT) or fasting insulinaemia at 13 weeks in chow or HFD-fed Sham, SubQ $\rightarrow$ Vis and Vis $\rightarrow$ Vis mice $(n=40)$. All cytokines in Fig. 6, except IL-6, predicted fasting insulinaemia (Fig. 7a); however, only TNF- $\alpha$ and IL-17 concentrations predicted both iAUC GTT and fasting insulinaemia (Fig. 7a-c). None of the cytokines measured at 4 weeks significantly predicted subsequent changes in glucose tolerance (data not shown).

Finally, to determine whether changes in AT gene expression influenced systemic cytokine concentrations, we examined the mRNA expression of Tnfa, Il6, Il17, Il12a, $\mathrm{Ccl}$, $\mathrm{Ccl} 4$ and $\mathrm{Ccl} 5$ in endogenous and grafted AT from our microarray data. Differences in plasma cytokine concentrations were not reflected in AT gene expression (Fig. 7d-j), and staining of SubQ $\rightarrow$ Vis and Vis $\rightarrow$ Vis grafts for F $4 / 80$, a marker of AT macrophages [36], did not reveal marked differences in macrophage content (ESM Fig. 5).

\section{Discussion}

Transplantation of inguinal, but not epididymal, AT uniquely and reproducibly protects mice against HFD-induced glucose intolerance and may reduce adiposity $[19,20]$. Here, SubQ $\rightarrow$ Vis transplantation improved glucose tolerance at 6 weeks, and was not explained by differences in adiposity, leptin, adiponectin or tissue-specific glucose uptake. Instead, SubQ $\rightarrow$ Vis transplantation reduced hepatic triacylglycerol accumulation and normalised gluconeogenic enzyme expression. Reduced hepatic triacylglycerol content in SubQ $\rightarrow$ Vis mice was not present at 6 weeks, however, suggesting that this occurs subsequent to improved glucose tolerance. SubQ $\rightarrow$ Vis transplantation also suppressed HFD-induced systemic inflammation: plasma concentrations of TNF- $\alpha$, IL-17, IL$12 p 70$, MCP-1/CCL2 and MIP-1 $\beta / C C L 4$ were all significantly reduced in SubQ $\rightarrow$ Vis mice, relative to Sham mice. Plasma IL-17 and MIP-1 $\beta$ concentrations were reduced in SubQ $\rightarrow$ Vis mice as early as 4 weeks post-transplantation, possibly preceding improvements in glucose tolerance. 
Fig. 6 (a-l) Plasma cytokine concentrations at 4 and 10 weeks post-transplantation. Cytokines displaying significant differences among groups at either 4 or 10 weeks are shown. Each graph shows the mean \pm SEM plasma cytokine concentration in chow (vertical striped bars), HFD-fed Sham (black bars), HFD-fed SubQ $\rightarrow$ Vis (white bars) and HFD-fed Vis $\rightarrow$ Vis (diagonally striped bars) mice. The remainder are shown in ESM Table 3. ${ }^{*} p<0.05$ vs Sham; ${ }^{\dagger} p<0.05$ vs $\mathrm{Vis} \rightarrow \mathrm{Vis}$
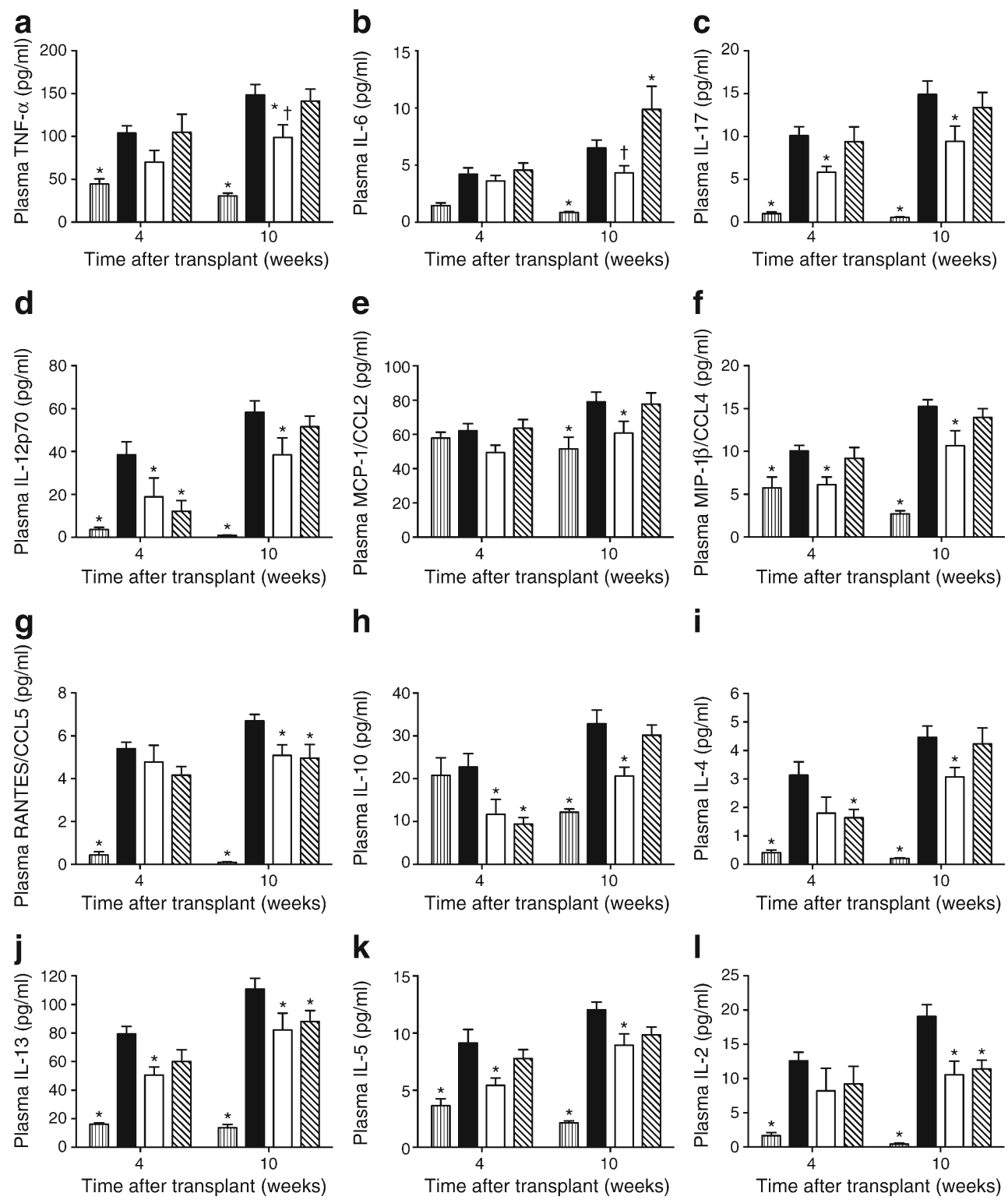

h

i


This study is the first to show anti-inflammatory effects of SubQ $\rightarrow$ Vis transplantation, although previous studies have suggested that Vis $\rightarrow$ Vis transplantation has proinflammatory effects [20, 37-39]. Immune cell composition differs between AT depots and is highly influenced by obesity [40]. In C57BL6/J mice, epididymal fat is skewed towards innate immunity, while the inguinal depot contains higher proportions of adaptive immune cells $(\mathrm{CD} 4+$ and $\mathrm{CD} 8+\mathrm{T}$ cells and $\mathrm{B}$ cells) [40]. Obesity is characterised by an increase in proinflammatory Th1 cells, particularly in VAT [35]. SubQ $\rightarrow$ Vis transplantation likely prevents HFD-induced glucose intolerance and IR by altering the balance of these cell types specifically in the intra-abdominal compartment, as SubQ $\rightarrow$ SubQ transplantation does not affect glucose tolerance [19, 20]. While further studies will be required to fully understand how this procedure affects immune cell populations, it will be important to consider migration of cells from donor AT to other tissues, including liver and spleen, following transplantation [41]. We were unable to relate differences in plasma cytokine concentrations to mRNA expression in grafted or endogenous AT (Fig. 7).

The early differences in IL-17 concentrations, and their relationship with glucose intolerance and insulinaemia, support a role for T helper 17 (Th17) cells in the pathogenesis of HFD-induced IR. Th17 populations expand in obesity and are a major source of proinflammatory IL-17 [42]. Th17 expansion depends on IL-6 and leptin in the AT microenvironment [43]. Genetic deletion [44] or antibody-mediated neutralisation [45] of IL-17 prevents IR, glucose intolerance and liver injury in HFD-fed mice. Human studies also implicate IL-17 in the pathogenesis of IR [46, 47]. IL-17 may link AT inflammation with hepatic 
Fig. 7 Relationships between plasma cytokine concentrations, glucose tolerance and insulinaemia. (a) Correlations between individual cytokines at 10 weeks post-transplantation and glucose tolerance or fasting insulinaemia at 13 weeks; $N=40$ mice (10 chow, 12 HFD Sham, 8 HFD SubQ $\rightarrow$ Vis and 10 HFD Vis $\rightarrow$ Vis). (b) Correlation between plasma TNF- $\alpha$ concentrations and iAUC GTT. Chow (white squares, $n=10$ ), HFD Sham (black circles, $n=12$ ), HFD SubQ $\rightarrow$ Vis (white circles, $n=8)$ and HFD Vis $\rightarrow$ Vis (black squares, $n=10$ ) mice are shown; Pearson $r=0.34, p=0.033$. (c) Correlation between plasma IL-17 concentrations and iAUC GTT; symbols are as for Fig. 7b; Spearman $r=0.34, p=0.032$. (d) Tnfa expression in grafted and endogenous AT by microarray. Normalised gene expression is provided in arbitrary units. (e-j) Expression of $I l 6(\mathbf{e})$; Ill7 (f); Ill2a (g); Ccl2 (h); Ccl4 (i); and $C c l 5$ (j); $* * p<0.01$ for comparison

\section{a}
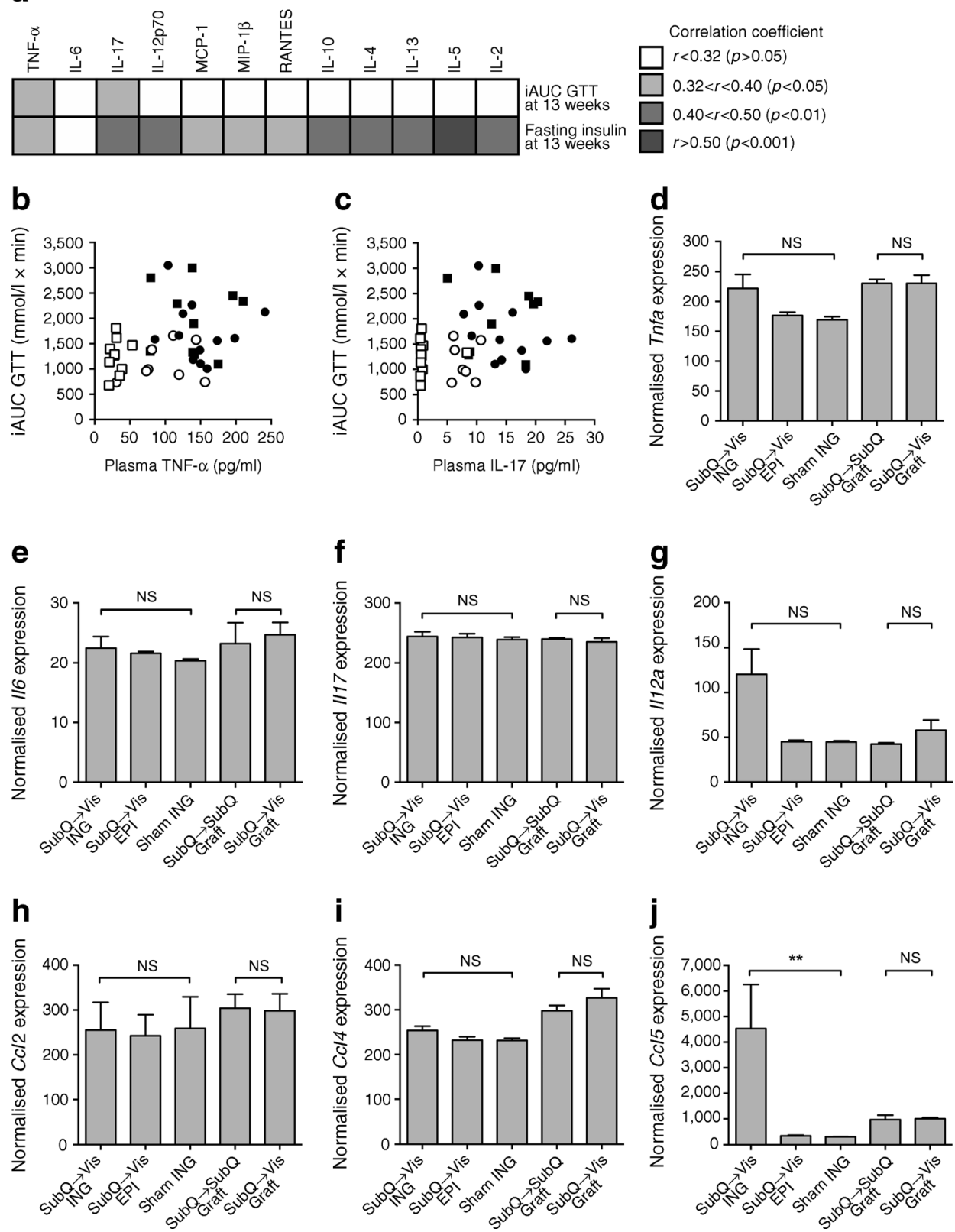

IR, as pre-treating human hepatocytes with IL-17 inhibited insulin-stimulated Akt phosphorylation and suppression of gluconeogenesis [16].

Beneficial effects of Vis $\rightarrow$ Vis transplantation (with caval drainage, not portal) on glucose tolerance have been reported at 4-8 weeks post-transplantation [18, 21, 39]. In the short term ( $\leq 6$ weeks), this additional VAT may reduce endogenous adiposity (Table 1), and protect against lipotoxicity (Fig. 5); but these effects are not sustained. Relative to Sham mice, Vis $\rightarrow$ Vis transplantation reduces HFDinduced hyperinsulinaemia, elevates plasma IL-6 concentrations, but does not prevent glucose intolerance or hepatic triacylglycerol accumulation.
Subcutaneous AT transplantation reproducibly prevents HFD-induced glucose intolerance in mice. While the mechanisms are incompletely understood, we have identified sustained anti-inflammatory effects of this procedure that predicted metabolic improvements. Future studies will be required to identify the cell type(s) responsible, and potential sites of intervention for the treatment and prevention of dietinduced glucose intolerance and IR.

Acknowledgements The authors would like to thank P. Lee, S. Grey and W. Kaplan (Garvan Institute) for their constructive comments on the manuscript, as well as the staff of the Garvan Institute Histopathology (A. Boulghourjian and A. Zaratzian) and Biological Testing Facilities. These 
results were presented at the 2014 Australian and New Zealand Obesity Society (ANZOS) Annual Scientific Meeting in Sydney, Australia.

Funding This work was supported by project (to MMS, SLH and AEB) and program (to DEJ and GJC) grants from the National Health and Medical Research Council (NHMRC) of Australia.

Duality of interest EK, SPB and MM are paid employees of Regeneus, Ltd. BRH is a nonexecutive director and paid consultant of Regeneus, Ltd. SPB, MM and BRH are shareholders in Regeneus Ltd. Regeneus Ltd did not provide funding for any of the experiments detailed in this manuscript. GAK, ZM and JRJ are employees of and shareholders in Genentech, Inc.

Contribution statement All authors made substantial contributions to the conception and design of the various aspects of the prospective studies or to the acquisition, analysis or interpretation of data. All authors also contributed to drafting the article or revising it critically for important intellectual content and have given final approval of the version to be published. MMS and SLH are responsible for the integrity of this work as a whole, including the study design, access to data, and the decision to submit and publish the manuscript.

\section{References}

1. Kissebah AH, Krakower GR (1994) Regional adiposity and morbidity. Physiol Rev 74:761-811

2. Carr DB, Utzschneider KM, Hull RL et al (2004) Intra-abdominal fat is a major determinant of the National Cholesterol Education Program Adult Treatment Panel III criteria for the metabolic syndrome. Diabetes 53:2087-2094

3. Bjorntorp P (1990) "Portal" adipose tissue as a generator of risk factors for cardiovascular disease and diabetes. Arteriosclerosis 10:493-496

4. Nielsen S, Guo Z, Johnson CM, Hensrud DD, Jensen MD (2004) Splanchnic lipolysis in human obesity. J Clin Invest 113:1582-1588

5. Fontana L, Eagon JC, Trujillo ME, Scherer PE, Klein S (2007) Visceral fat adipokine secretion is associated with systemic inflammation in obese humans. Diabetes 56:1010-1013

6. Manolopoulos KN, Karpe F, Frayn KN (2010) Gluteofemoral body fat as a determinant of metabolic health. Int $\mathrm{J}$ Obes (Lond) 34:949-959

7. Manolopoulos KN, Karpe F, Frayn KN (2012) Marked resistance of femoral adipose tissue blood flow and lipolysis to adrenaline in vivo. Diabetologia 55:3029-3037

8. Frayn KN (2002) Adipose tissue as a buffer for daily lipid flux. Diabetologia 45:1201-1210

9. Pinnick KE, Nicholson G, Manolopoulos KN et al (2014) Distinct developmental profile of lower-body adipose tissue defines resistance against obesity-associated metabolic complications. Diabetes 63:3785-3797

10. Swarbrick MM (2014) A lifetime on the hips: programming lowerbody fat to protect against metabolic disease. Diabetes 63:35753577

11. Xu H, Barnes GT, Yang Q et al (2003) Chronic inflammation in fat plays a crucial role in the development of obesity-related insulin resistance. J Clin Invest 112:1821-1830

12. Sun S, Ji Y, Kersten S, Qi L (2012) Mechanisms of inflammatory responses in obese adipose tissue. Annu Rev Nutr 32:261-286

13. Weisberg SP, McCann D, Desai M, Rosenbaum M, Leibel RL, Ferrante AW Jr (2003) Obesity is associated with macrophage accumulation in adipose tissue. J Clin Invest 112:1796-1808
14. Lumeng CN, Bodzin JL, Saltiel AR (2007) Obesity induces a phenotypic switch in adipose tissue macrophage polarization. J Clin Invest 117:175-184

15. Osborn O, Olefsky JM (2012) The cellular and signaling networks linking the immune system and metabolism in disease. Nat Med 18: 363-374

16. Fabbrini E, Cella M, McCartney SA et al (2013) Association between specific adipose tissue CD4+ T-cell populations and insulin resistance in obese individuals. Gastroenterology 145(366-374): e361-e363

17. Goldfine AB, Fonseca V, Jablonski KA, Pyle L, Staten MA, Shoelson SE (2010) The effects of salsalate on glycemic control in patients with type 2 diabetes: a randomized trial. Ann Intern Med 152:346-357

18. Konrad D, Rudich A, Schoenle EJ (2007) Improved glucose tolerance in mice receiving intraperitoneal transplantation of normal fat tissue. Diabetologia 50:833-839

19. Hocking SL, Chisholm DJ, James DE (2008) Studies of regional adipose transplantation reveal a unique and beneficial interaction between subcutaneous adipose tissue and the intra-abdominal compartment. Diabetologia 51:900-902

20. Tran TT, Yamamoto Y, Gesta S, Kahn CR (2008) Beneficial effects of subcutaneous fat transplantation on metabolism. Cell Metab $7: 410-420$

21. Foster MT, Shi H, Softic S, Kohli R, Seeley RJ, Woods SC (2011) Transplantation of non-visceral fat to the visceral cavity improves glucose tolerance in mice: investigation of hepatic lipids and insulin sensitivity. Diabetologia 54:2890-2899

22. Foster MT, Softic S, Caldwell J, Kohli R, de Kloet AD, Seeley RJ (2013) Subcutaneous adipose tissue transplantation in diet-induced obese mice attenuates metabolic dysregulation while removal exacerbates it. Physiol Rep 1:e00015

23. Hoehn KL, Turner N, Swarbrick MM et al (2010) Acute or chronic upregulation of mitochondrial fatty acid oxidation has no net effect on whole-body energy expenditure or adiposity. Cell Metab 11:70-76

24. Crosson SM, Khan A, Printen J, Pessin JE, Saltiel AR (2003) PTG gene deletion causes impaired glycogen synthesis and developmental insulin resistance. J Clin Invest 111:1423-1432

25. Lau SM, Cha KM, Karunatillake A et al (2013) Beta-cell ARNT is required for normal glucose tolerance in murine pregnancy. PLoS One 8:e77419

26. Reich M, Liefeld T, Gould J, Lerner J, Tamayo P, Mesirov JP (2006) GenePattern 2.0. Nat Genet 38:500-501

27. Subramanian A, Tamayo P, Mootha VK et al (2005) Gene set enrichment analysis: a knowledge-based approach for interpreting genome-wide expression profiles. Proc Natl Acad Sci U S A 102: $15545-15550$

28. Mootha VK, Lindgren CM, Eriksson KF et al (2003) PGC-1 $\alpha$ responsive genes involved in oxidative phosphorylation are coordinately downregulated in human diabetes. Nat Genet 34:267-273

29. Munoz S, Franckhauser S, Elias I et al (2010) Chronically increased glucose uptake by adipose tissue leads to lactate production and improved insulin sensitivity rather than obesity in the mouse. Diabetologia 53:2417-2430

30. Clark RI, Tan SW, Pean CB et al (2013) MEF2 is an in vivo immune-metabolic switch. Cell 155:435-447

31. Reed BD, Charos AE, Szekely AM, Weissman SM, Snyder M (2008) Genome-wide occupancy of SREBP1 and its partners NFY and SP1 reveals novel functional roles and combinatorial regulation of distinct classes of genes. PLoS Genet 4:e1000133

32. Patsouris D, Li PP, Thapar D, Chapman J, Olefsky JM, Neels JG (2008) Ablation of CD11c-positive cells normalizes insulin sensitivity in obese insulin resistant animals. Cell Metab 8:301-309

33. Jiao P, Chen Q, Shah S et al (2009) Obesity-related upregulation of monocyte chemotactic factors in adipocytes: involvement of 
nuclear factor-kappaB and c-Jun NH2-terminal kinase pathways. Diabetes 58:104-115

34. Yepuru M, Eswaraka J, Kearbey JD et al (2010) Estrogen receptor\{beta\}-selective ligands alleviate high-fat diet- and ovariectomyinduced obesity in mice. J Biol Chem 285:31292-31303

35. Sell H, Habich C, Eckel J (2012) Adaptive immunity in obesity and insulin resistance. Nat Rev Endocrinol 8:709-716

36. Bassaganya-Riera J, Misyak S, Guri AJ, Hontecillas R (2009) PPAR gamma is highly expressed in F4/80(hi) adipose tissue macrophages and dampens adipose-tissue inflammation. Cell Immunol 258:138-146

37. Ohman MK, Shen Y, Obimba CI et al (2008) Visceral adipose tissue inflammation accelerates atherosclerosis in apolipoprotein Edeficient mice. Circulation 117:798-805

38. Nov O, Shapiro H, Ovadia $\mathrm{H}$ et al (2013) Interleukin-1beta regulates fat-liver crosstalk in obesity by auto-paracrine modulation of adipose tissue inflammation and expandability. PLoS One 8:e53626

39. Rytka JM, Wueest S, Schoenle EJ, Konrad D (2011) The portal theory supported by venous drainage-selective fat transplantation. Diabetes 60:56-63

40. Caspar-Bauguil S, Cousin B, Galinier A et al (2005) Adipose tissues as an ancestral immune organ: site-specific change in obesity. FEBS Lett 579:3487-3492
41. Paz-Filho G, Mastronardi CA, Parker BJ et al (2013) Molecular pathways involved in the improvement of non-alcoholic fatty liver disease. J Mol Endocrinol 51:167-179

42. Winer S, Paltser G, Chan Y et al (2009) Obesity predisposes to Th17 bias. Eur J Immunol 39:2629-2635

43. Chuang HC, Sheu WH, Lin YT et al (2014) HGK/MAP4K4 deficiency induces TRAF2 stabilization and Th17 differentiation leading to insulin resistance. Nat Commun 5:4602

44. Zuniga LA, Shen WJ, Joyce-Shaikh B et al (2010) IL-17 regulates adipogenesis, glucose homeostasis, and obesity. J Immunol 185: 6947-6959

45. Harley IT, Stankiewicz TE, Giles DA et al (2014) IL-17 signaling accelerates the progression of nonalcoholic fatty liver disease in mice. Hepatology 59:1830-1839

46. Jagannathan-Bogdan M, McDonnell ME, Shin H et al (2011) Elevated proinflammatory cytokine production by a skewed T cell compartment requires monocytes and promotes inflammation in type 2 diabetes. J Immunol 186:1162-1172

47. Goossens GH, Blaak EE, Theunissen R et al (2012) Expression of NLRP3 inflammasome and T cell population markers in adipose tissue are associated with insulin resistance and impaired glucose metabolism in humans. Mol Immunol $50: 142-149$ 